\title{
Bed Load Transport Modelling Using Kinetic Theory
}

\author{
Václav Matoušek ${ }^{1}$ and Štěpán Zrostlík ${ }^{1}$ \\ ${ }^{1}$ Czech Technical University in Prague, Civil Engineering, 16629 Prague 6, Czech Republic
}

\begin{abstract}
Intense transport of bed load is associated with highconcentrated sediment-laden flow over a plane mobile bed at high bed shear. Typically, the flow exhibits a layered internal structure in which a vast majority of sediment grains is transported through a collisional layer above the bed. Our investigation focuses on steady uniform open-channel flow with a developed collisional transport layer and combines modelling and experiment to relate integral quantities, as the discharge of solids, discharge of mixture, and flow depth with the longitudinal slope of the bed and the internal structure of the flow above the bed. In the paper, flow with the internal structure described by linear vertical distributions of granular velocity and concentration across the collisional layer is analyzed by a model based on the classical kinetic theory of granular flows. The model predicts the total discharge, the discharge of sediment, and the flow depth for given (experimentally determined) bed slope and thickness of collisional layer. The model also predicts whether the intefacial dense layer develops between the bed and the collisional layer and how thick it is. Model predictions are compared with results of intense bed-load experiment carried out for lightweight sediment in our laboratory tilting flume.
\end{abstract}

\section{Introduction}

For intense bed load transport, collisional interactions of transported sediment grains are typical and they significantly affect behaviour of flow carrying the sediment above a plane mobile bed at high bed shear (the upper-stage bed regime). The flow exhibits a layered structure in which a vast majority of grains is transported through a collisional transport layer. If the bed shear stress exerted by the flow is very high, a sliding dense layer tends to develop between the collisional layer and the bed. In the dense layer, grains remain in virtually permanent contact and slide over each other rather than collide with each other. Typically, the collisional layer dominates the internal structure of the flow. Appropriate modelling of friction and transport in the layered structure of the flow is crucial for prediction ability of a bed-load transport model. So far, collisional mechanisms are poorly understood and hence modelling approaches are seldom sufficiently accurate.

One of the appropriate approaches to modelling of flows dominated by granular collision seems to be the kinetic theory of granular flows. In general, kinetic-theory based models for contact-load transport in open-channel flow enable a prediction of relevant flow quantities in a layered pattern of the flow. Model predictions include integral flow 
quantities (discharges of solids and mixture, flow depth) and simplified distributions of solids concentration and velocity. The existing models differ mainly in assumptions taken to calculate granular flows under particular studied conditions, e.g. [1-3]. In this paper, we aim on formulating a simple kinetic-theory-based model to evaluate flow conditions observed in our intense-bed-load experiment in a laboratory tilting flume.

\section{Principles of kinetic-theory based modelling}

Classical kinetic theory (CKT) considers sheared granular bodies, in which grains are supported exclusively by mutual binary collisions. Constitutive relations are formulated for local grain stresses (normal and shear) and for a balance of grain fluctuation energy in the collisional regime.

\subsection{Constitutive relations}

The local shear-induced granular normal stress, $\sigma_{s}$, is related to the local volumetric concentration of grains, $c$, the local granular temperature, $T$,

$$
\sigma_{s}=4 \cdot \rho_{s} \cdot f_{\sigma} \cdot c \cdot G \cdot T
$$

where $\rho_{s}=$ density of grains, $d=$ grain size, and $G, f_{\sigma}=$ concentration-related functions defined as

$$
\begin{aligned}
& G=c \cdot \frac{2-c}{2 \cdot(1-c)^{3}} \\
& f_{\sigma}=\frac{1+e}{2}+\frac{1}{4 \cdot G}
\end{aligned}
$$

where $e=$ the coefficient of wet restitution.

The local shear-induced granular shear stress, $\tau_{s}$, is also related to $c$ and $T$ at any vertical position $y$ above the bed. Moreover, $\tau_{s}$ is related to the local strain rate $\gamma$, i.e. the distribution of longitudinal velocity of grains $u(\gamma=\mathrm{d} u / \mathrm{d} y)$,

$$
\tau_{s}=\rho_{s} \cdot f_{\tau} \cdot c \cdot G \cdot \sqrt{T} \cdot \gamma \cdot d
$$

where concentration-related function

$$
f_{\tau}=\frac{8}{5 \cdot \sqrt{\pi}} \cdot\left(\frac{1+e}{2}+\frac{\pi}{32} \cdot \frac{[5+2 \cdot(1+e) \cdot(3 \cdot e-1) \cdot G] \cdot[5+4 \cdot(1+e) \cdot G]}{\left[24-6 \cdot(1-e)^{2}-5 \cdot\left(1-e^{2}\right)\right] \cdot G^{2}}\right)
$$

Another constitutive relation expresses the balance of the particle collisional fluctuation energy. It requires that the gradient of the vertical component of the flux of particle fluctuation energy balances the net rate of production of fluctuation energy per unit volume of the mixture [4]. The relation is composed of three terms. The first term represents the diffusion of fluctuation energy, the second term the production of energy due to shearing, and the third term represents the rate of collisional dissipation, i.e. the fluctuation energy dissipated by interparticle collisions [5]. For our conditions, the diffusion term can be neglected [6] and the kinetic-energy relation becomes an additional equation relating the granular shear stress with the granular temperature and the strain rate,

$$
\tau_{s}=\frac{24}{\sqrt{\pi}} \cdot \rho_{s} \cdot c \cdot G \cdot(1-e) \cdot \frac{\sqrt{T^{3}}}{\gamma \cdot d}
$$




\subsection{Momentum balance equations}

Alternative equations relating the distribution of the local concentration with the distributions of the granular stresses are based on the principle of momentum balance. In gravity-driven solid-liquid flow with a free surface, the force balance between the driving force and the resisting force assumes that the total shear stress, $\tau_{e}$ (composed of the granular component, $\tau_{s}$, and the liquid component, $\tau_{f}$ ) at each vertical position $y$ balances the longitudinal component of the weight of overlaying burden of liquid and solids,

$$
\tau_{e}=g \cdot \sin \omega \cdot \int_{y}^{H}\left[\rho_{s} \cdot c+\rho_{f} \cdot(1-c)\right] \cdot d y
$$

in which $\rho_{f}=$ density of liquid, $g=$ gravitational acceleration, $\omega=$ angle of longitudinal slope of bed, and $H=$ total flow depth.

The granular normal stress balances the normal component of the submerged weight of grains above $y$,

$$
\sigma_{s}=\left(\rho_{s}-\rho_{f}\right) \cdot g \cdot \cos \omega \cdot \int_{y}^{H} c \cdot d y
$$

It follows from re-arrangements of Eqs. 7-8 that

$$
\tau_{e}=\sigma_{s} \cdot \tan \omega+\rho_{f} \cdot g \cdot \sin \omega \cdot(H-y)
$$

where the first term on the right-hand side of Eq. 9 is the granular component, $\tau_{s}$, of the total shear stress and the second term is the liquid component, $\tau_{f}$.

\section{Modelling of intense bed load}

A modelling approach is discussed which enables to predict characteristics of steady uniform open-channel flow carrying a large amount of colliding sediment (intense bedload). The approach is based on the classical kinetic theory, considers a layered structure of the sediment-laden flow and employs conditions at layer interfaces to evaluate mutual relations among the flow slope, depth, the thickness of the layers and flow rates of both the sediment and sediment-water mixture. In the discussed model, the dense limit condition (e.g. [3]) is not assumed at the bottom of the collisional transport layer because our experiments indicated that for studied flow conditions the local concentration at the bottom of the collisional layer varied considerable with the bed shear stress. At low bed shear stresses, it reached values too low to satisfy the dense limit condition.

\subsection{Studied conditions}

- Gravity-driven open-channel flow, steady-state uniform flow.

- Flow over mobile bed at upper-stage plane bed regime (high bed shear).

- Transported sediment grains supported exclusively by mutual contacts, no turbulent suspension.

- Broad range of bed slopes, flows depths, sediment flow rates, and total flow rates.

\subsection{Internal structure of flow}

It is typical for bed load flows that local concentrations and velocities of grains span a broad range of values over the thickness of the collisional layer. Visual observations of sediment-laden flows tested in a tilting flume of the Czech Technical University in Prague revealed a layered character of intense bed-load [7], see Figure 1 for lightweight sediment 
TLT50 (the grain size of $5.4 \mathrm{~mm}$, the density of $1368 \mathrm{~kg} / \mathrm{m}^{3}$ ). The following distinct layers were identified: the bed (stationary deposit with the upper 0-boundary in Figure 1), the dense sliding layer (DL with the upper d-boundary), the collisional layer (CL with the upper c-boundary), and the water layer (WL with water surface at the top of the plot in Figure 1). Grains do not move in the bed, they slide over each other in the DL, and collide with each other in the CL. The WL is grain free.

The thickness of individual layers varied with the bed Shields parameter $\theta_{0}$. No dense layer developed at low values of Shields parameter. An analysis of measured discharges suggested that the local concentration at the bottom of collisional layer, $c_{d}$, was smaller than the bed concentration, $c_{0}$, and varied with $\theta_{0}$ until a certain maximum value typical for bed was reached [7], see also plots in Figure 1 for two different values of the integral-quantity based Shields parameter at top of bed, $\theta$.

The existence of the individual layers and their variation in thickness with the boundary shear stress must be taken into account in the model.
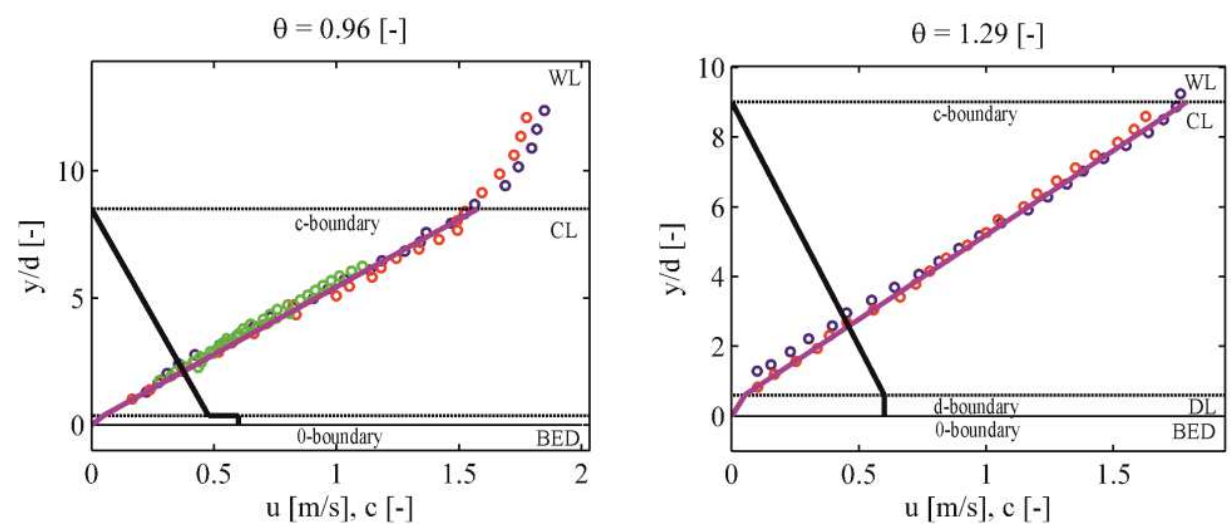

Fig. 1. Positions of interfaces, velocity and concentration profiles in layered-structure flow carrying TLT50-grains. Legend: circle - measurement of local velocity by different measuring techniques $($ green $=$ ADVP, red $=$ UVP, blue $=$ Pitot tube $)$; horizontal lines - interfaces between layers $($ bed, DL $=$ dense layer, $\mathrm{CL}=$ collisional layer, $\mathrm{WL}$ - water layer); thick lines - velocity profile (magenta) and concentration profile (black) [7].

\subsection{Model features}

Semi-empirical transport formulae for bed load relate the solids discharge with the shear stress at the top of the mobile bed. A kinetic-theory based model can serve the same purpose by relating the granular shear stress at the bottom of the collisional layer with relevant flow quantities responsible for the solids discharge. Furthermore, an incorporation of the momentum balance equations allows to capture the layered structure of the flow and to identify positions of the layer interfaces. For chosen (e.g. experimentally determined) input quantities, the model does not require the classical law of the wall to relate the total discharge with the flow depth.

In the presented model, the constitutive relations of the classical kinetic theory are employed to describe granular flow conditions at the bottom of the CL, where the local concentration is supposed to vary with the boundary shear stress. The constitutive relations also predict the slope of the linear profile of solids velocity in the CL and hence they determine the local velocity $u_{c}$ at the top of the CL. The momentum balances are combined with the shear-to-normal stress ratios at the relevant interfaces to determine positions of the 
boundaries in the layered flow structure. The discharges of solids and mixture are obtained through integration of the velocities and concentrations over the flow depth.

\subsection{Model assumptions}

- Distribution of velocity and concentration linear in the CL, concentration distribution uniform and solids velocity negligible in the DL. Local concentration zero at the top of CL (c-boundary).

- Negligible fluid stress at the d-boundary and at the 0-boundary.

- No side-wall effect.

- No local slip between grain and liquid in the CL and DL.

- The diffusive term of the energy-balance relation is negligible at the d-boundary (and 0boundary).

\subsection{Model equations}

The model is composed of the following set of equations (constitutive relations, momentum balances, and closures). At the bottom of the collisional layer (the d-boundary at high $\theta_{0}$, the 0 -boundary at low $\theta_{0}$ ) the constitutive relations (classical kinetic theory) for the shear induced granular stresses read

$$
\begin{aligned}
& \sigma_{s, d}=4 \cdot \rho_{s} \cdot f_{\sigma, d} \cdot c_{d} \cdot G_{d} \cdot T_{d} \\
& \tau_{s, d}=\rho_{s} \cdot f_{\tau, d} \cdot c_{d} \cdot G_{d} \cdot \sqrt{T_{d}} \cdot \gamma_{d} \cdot d
\end{aligned}
$$

and from the energy balance with negligible diffusion term

$$
\tau_{s, d}=\frac{24}{\sqrt{\pi}} \cdot \rho_{s} \cdot c_{d} \cdot G_{d} \cdot\left(1-e_{d}\right) \cdot \frac{\sqrt{T_{d}^{3}}}{\gamma_{d} \cdot d}
$$

The local coefficient of restitution at the bottom of the CL, $e_{d}$, equals (e.g. [3])

$$
e_{d}=\varepsilon-62.1 \cdot \frac{\mu_{f} \cdot(1+\varepsilon)}{\rho_{s} \cdot \sqrt{T_{d}} \cdot d}
$$

in which $\varepsilon=$ effective coefficient of dry collisional restitution, $\mu_{f}=$ dynamic viscosity of fluid. The two shear-stress relations (Eqs. 11-12) combined give

$$
\gamma_{d}=\sqrt{\frac{24 \cdot\left(1-e_{d}\right) \cdot T_{d}}{\sqrt{\pi} \cdot f_{\tau, d} \cdot d^{2}}}
$$

The linear distribution across the CL leads to

$$
u_{c}=u_{d}+\gamma_{d} \cdot\left(y_{c}-y_{d}\right)
$$

At the d-boundary, the granular stresses are related through the friction coefficient

$$
\beta_{d}=\tau_{s, d} / \sigma_{s, d}
$$

At the bottom of the flow, the Coulomb yield criterion requires

$$
\beta_{0}=\tau_{s, 0} / \sigma_{s, 0}
$$

The momentum balance equations based on Eqs. 7-9 relate the local stresses with the positions of the boundaries (d-boundary, c-boundary, and water surface) above the top of the bed (0-boundary),

$$
\begin{aligned}
& \sigma_{s, \mathrm{~d}}=\left(\rho_{s}-\rho_{f}\right) \cdot c_{d} \cdot g \cdot \sin \omega \cdot\left(y_{c}-y_{d}\right) / 2 \\
& \sigma_{s, 0}=\sigma_{s, d}+\left(\rho_{s}-\rho_{f}\right) \cdot c_{0} \cdot g \cdot \sin \omega \cdot y_{d}
\end{aligned}
$$




$$
\begin{gathered}
\tau_{e, 0}=\tau_{e, d}+\left(\sigma_{s, 0}-\sigma_{s, d}\right) \cdot \tan \omega+\rho_{f} \cdot g \cdot \sin \omega \cdot y_{d} \\
\tau_{e, d}=\tau_{e, c}+\sigma_{s, d} \cdot \tan \omega+\rho_{f} \cdot g \cdot \sin \omega \cdot\left(y_{c}-y_{d}\right) \\
\tau_{e, c}=\tau_{f, c}=\rho_{f} \cdot g \cdot \sin \omega \cdot\left(H-y_{c}\right)
\end{gathered}
$$

Linear distributions of $u$ and $c$ across the CL (with assumed $c_{c}=0, u_{d}=0$ ) are combined with the earlier determined positions of the boundaries to give the sediment discharge,

$$
q_{s}=\frac{c_{d} \cdot u_{c} \cdot\left(y_{c}-y_{d}\right)}{6}
$$

If the assumptions of no slip in the CL and of the uniform velocity distribution in the WL are taken, then the total discharge (mixture of sediment and liquid) is

$$
q_{m}=\frac{u_{c} \cdot\left(y_{c}-y_{d}\right)}{2}+u_{c} \cdot\left(H-y_{c}\right)
$$

The average spatial volumetric concentration of sediment in the flow cross section is

$$
C_{v i}=\frac{c_{0} \cdot y_{d}+c_{d} / 2 \cdot\left(y_{c}-y_{d}\right)}{H}
$$

The dimensionless total shear stress (Shields parameter) at the bed surface,

$$
\theta_{0}=\left(C_{v}-\frac{\rho_{f}}{\rho_{s}-\rho_{f}}\right) \cdot \frac{H}{d} \cdot \tan \omega
$$

\subsection{Inputs/outputs}

In general, there are 4 mutually related major quantities characterizing the sediment-laden flow: the longitudinal slope of bed, $\omega$, the flow depth, $H$, the sediment discharge, $q_{s}$, and the mixture discharge, $q_{m}$. To be able to compare model predictions with experimental results of the TLT50-test, we use the measured bed slope and the measured thickness of $\mathrm{CL}, y_{c}-y_{d}$, as inputs and predict the flow depth and the two discharges. Alternatively, the model can consider the flow depth as an input and to predict the thickness of the CL.

Additional model outputs are the position of the top of the DL, $y_{d}$, the velocity at the top of the CL, $u_{c}$, and the granular-stress ratio at the bottom of the CL, $\beta_{\mathrm{d}}$. To initialize the model calculation, experimental values of $c_{d}$ and $\left(y_{c}-y_{d}\right)$ are used together with additional model inputs - properties of solids $\left(\rho_{\mathrm{s}}, d\right)$ and fluid $\left(\rho_{\mathrm{f}}, \mu_{\mathrm{f}}\right)$, the coefficient of internal friction at the top of bed, $\beta_{0}$, the bed concentration $\mathrm{c}_{0}$, and the dry restitution coefficient, $\varepsilon$.

\section{Discussion of results}

Figure 2 shows that the experimental local concentration at the bottom of the CL is quite sensitive to the bed Shields parameter $\theta_{0}$ and exhibits low values at flow conditions represented by the low Shields parameter. In the model, this variability in $c_{d}$ is employed to predict trends in developments of the thickness of the sliding dense layer $y_{d}$ (Figure 3 ) and of the velocity at the top of the collisional layer $u_{c}$ (Figure 4). The trends for both $u_{c}$ (calculated using Eqs. 10-15 and 18) and $y_{d}$ (obtained from Eqs. 16-17, 19-20) are captured well. Visual observations detected a presence of the dense layer at $\theta_{0}>0.9$ [7] and the model prediction of $y_{d}$ agrees with the observations (Figure 3). The experimental values of $u_{c}$ increased with $\theta_{0}$ and the sensitivity of $u_{c}$ on $\theta_{0}$ was much stronger at low $\theta_{0}$ than at high $\theta_{0}$. In Figure 4 , the parity plot indicates that the agreement between the measured and predicted $u_{c}$ is very similar in the entire range of $\theta_{0}$. The model predictions are for $\beta_{0}=0.6$, $c_{0}=0.55$, and $\varepsilon=0.75$. 

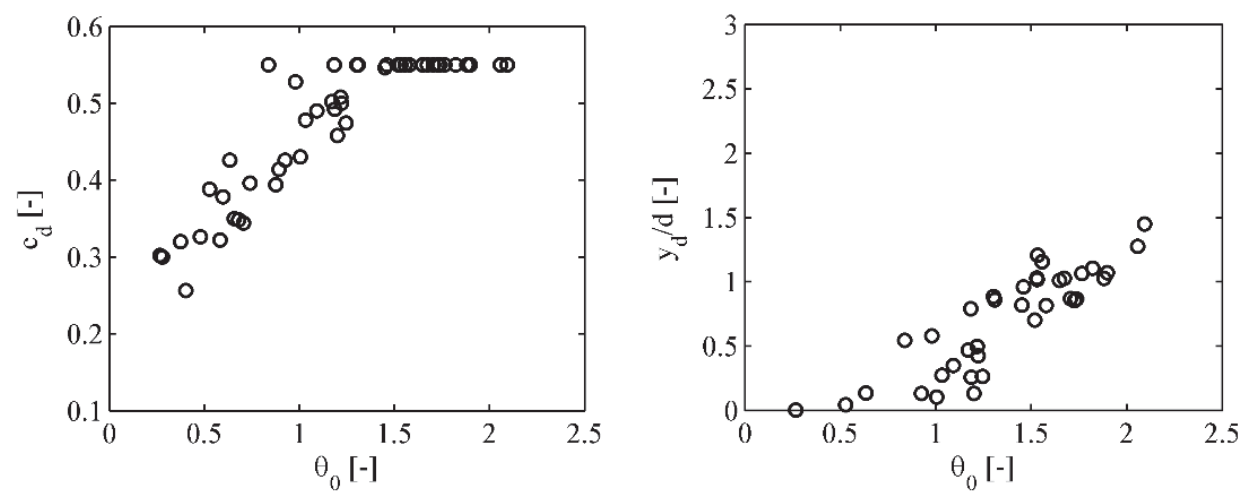

Fig. 2. (Left panel): Experimentally determined local concentration at bottom of collisional layer sensitive to bed Shields parameter (TLT50-experiments).

Fig. 3. (Right panel): Predicted relative thickness of dense layer (layer thickness divided by grain size) sensitive to bed Shields parameter.
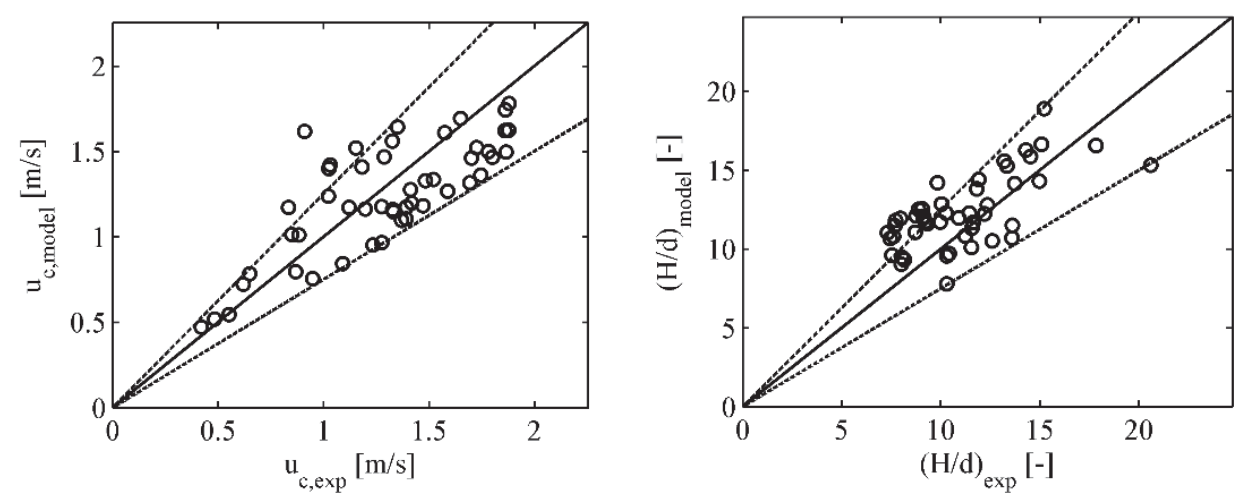

Fig. 4. (Left panel): Parity plot for experimental and predicted velocity at top of collisional layer (TLT50-tests). Legend: lines of perfect fit and of \pm 25 per cent deviation.

Fig. 5. (Right panel): Parity plot for experimental and predicted relative depth of flow (flow depth divided by grain size) (TLT50-tests). Legend: lines of perfect fit and of \pm 25 per cent deviation.

The flow depth $H$ (calculated by Eqs. 21-22) is also predicted well except for the lowest flow depths (Figure 5). The predicted sediment discharge $q_{s}$ (Eq. 23) deviates from the experimental values at the highest Shields parameters, where the low predicted values are a result of the model assumption $c_{c}=0$, which is inaccurate if the top of the collisional layer tends to the water surface. The ratio of two average cross-sectional concentrations is evaluated in Figure 7. The delivered concentration of sediment $C_{v d}=q_{s} / q_{m}$ (Eqs. 23-24) and its value must be smaller than the corresponding value of the spatial volumetric concentration $C_{v i}$ (Eq. 25), which is the case in Figure 7. 

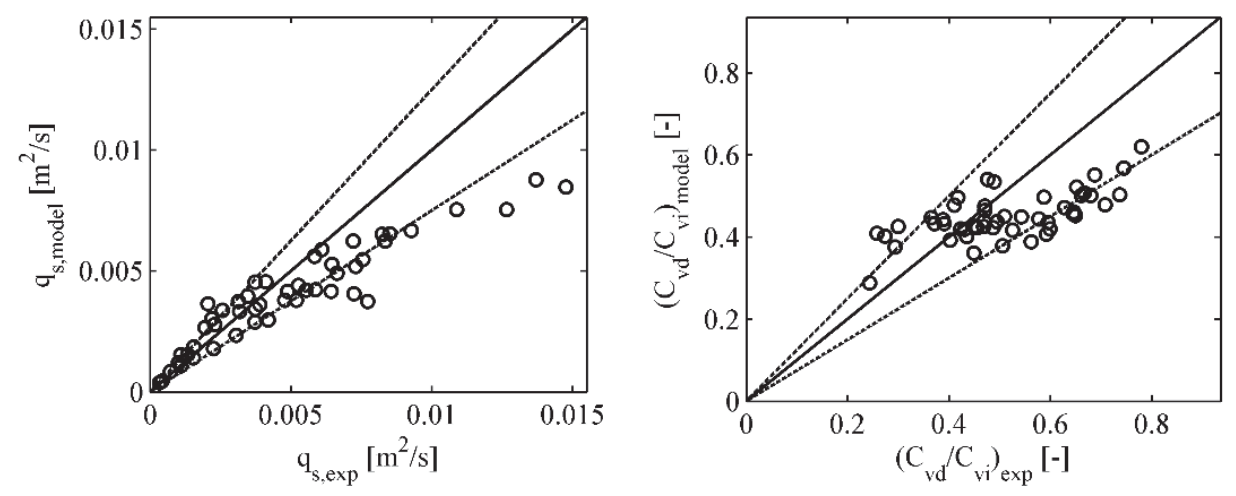

Fig. 6. (Left panel): Parity plot for experimental and predicted discharge of sediment (TLT50-tests). Legend: lines of perfect fit and of \pm 25 per cent deviation.

Fig. 7. (Right panel): Parity plot for experimental and predicted ratio of delivered concentration and spatial concentration of sediment (TLT50-tests). Legend: lines of perfect fit and of \pm 25 per cent deviation.

\section{Conclusions}

In the presented kinetic-theory based model, constitutive relations of the classical kinetic theory allow to predict the discharges of sediment and mixture, the flow depth and the thickness of the sliding dense layer in flow carrying intense bed load at conditions observed in a tilting-flume experiment. The model takes simple assumptions for interfaces of a multilayer structure of the observed sediment-laden flow at high bed shear. It applies the constitutive relations to flow conditions at the bottom of the collisional transport layer, where the experiment indicates a considerable variation of the local concentration of sediment with the local granular stress.

A comparison with the experimental results suggests that the model reasonably predicts flow rates of both sediment and mixture. For given bed slope and information about the collisional layer (the local concentrations at the bottom and at the top of the collisional layer, the thickness of the collisional layer), the model also predicts the flow depth and the thickness of the interfacial dense layer which links the collisional layer with the bed. The predictions are again in a reasonable agreement with the experimental observations.

The research has been supported by the Czech Science Foundation through the grant project No. 1621421S.

\section{References}

1. H. Capart, L. Fraccarollo, Geophys. Res. Lett. 38, L20402 (2011)

2. B. Spinewine, H. Capart, J. Fluid Mech. 731, 579-613 (2013)

3. D. Berzi, L. Fraccarollo, Geophys. Phys. Fluids 25, 106601 (2013)

4. J.T. Jenkins, D.M. Hanes, J. Fluid Mech. 370, 29-52 (1998)

5. A. Armanini, H. Capart, L. Fraccarollo, M. Larcher, J. Fluid Mech. 532, 269-319 (2005)

6. V. Matoušek, Š. Zrostlík, J. Hydrol. Hydromech., 66, in press (2018)

7. V. Matoušek, V. Bareš, J. Krupička, T. Picek, Š. Zrostlík, River Flow 2016 (IAHR, Saint Louis, 2016) 